Article

\title{
Development of Navigator Behavior Models for the Evaluation of Collision Avoidance Behavior in the Collision-Prone Navigation Environment
}

\author{
Jeong-Bin Yim ${ }^{1}$, Deuk-Jin Park ${ }^{2, *}$ and Ik-Hyun Youn ${ }^{3}$ \\ 1 Division of Navigation Sciences, Korea Maritime and Ocean University, Busan 49112, Korea \\ 2 Department of Maritime Transportation System, Mokpo National Maritime University, Mokpo 58628, Korea \\ 3 Division of Navigation \& Information Systems, Mokpo National Maritime University, Mokpo 58628, Korea \\ * Correspondence: pdj@mmu.ac.kr; Tel.: +82-61-240-7156
}

Received: 1 June 2019; Accepted: 30 July 2019; Published: 1 August 2019

check for updates

\begin{abstract}
In order to prevent ship collisions, it is important to understand the behavior of navigators that leads to these collisions. The main cause of marine accidents in the Republic of Korea is attributed to navigator error, particularly in collisions. Hence, reducing navigator error is a key issue that needs to be addressed to prevent accidents. However, the lack of objective measure to quantify navigator error remains a challenge. The purpose of this study is to develop an objective identification of a navigator's behavior in a collision encountering situation. Two behavior models for the success and failure of collision avoidance are developed by collecting participants' actions, using a ship maneuvering simulator within a given scenario. These maneuvering behavior models are validated in terms of their discrimination powers. The results show that maneuvering behavior is clearly identified in the data processing and model development phases. The proposed behavior models are expected to provide a better understanding of how navigators behave to help reduce collision accidents.
\end{abstract}

Keywords: maritime accidents; maneuver behavior identification; ship collision; collision avoidance; ship maneuvering simulator

\section{Introduction}

Understanding how navigators behave when they are involved in a ship collision is crucial for preventing collision accidents caused by human error, which remains a major cause of shipping incidents [1-3]. In relation to this, behavior models of navigators who operate in complex, high-risk domains are of great value because of high losses due to navigator failure $[4,5]$. To ensure the safety of maritime transportation, scientific measures must be taken to respond to and prevent various types of maritime accidents, instead of having ambiguous anticipations of possible human errors [6-8]. Although international rules have been reinforced by International Maritime Organization (IMO) to avoid navigator error, the accident caused by human error continues to this day [3,8]. Recently, the causes of accidents were induced by a model to assist with accident prevention, and the concept of accident causes found in the researchers' models was explained and discussed; however, none is yet generally accepted [9]. Human error is continually quoted as the source of an accident. However, the solution is thought to change its role in people or systems, and it is not really simple. Human error must link human behavior through the process [10]. Based on a recent assessment [11], navigator error was involved in $79 \%$ of accidents in the Republic of Korea over the last five years, and inappropriate behavior accounted for $49 \%$ of all maritime accidents [12]. Although most maritime accidents were suspected to be related to navigator error, evidence of navigator error has not yet been explored objectively [13]. Most existing assessments of a navigator's maneuvering behavior depend on indirect 
data collection methods, such as diaries, surveys, and questionnaires [14-16]. For example, Cordon et al. used a sample of 141 officers to investigate human errors of navigators via a survey of the importance of a navigator's behavior [17], and classify ship bridge work as a criterion for situational awareness for preventing navigator's error from occurring [18]. The use of such indirect approaches entails a subjective limitation for accurate observation of the maneuvering behavior of navigators during navigation. This limitation could be addressed using more objective data collection measures of a navigator's behavior. In relation to this, the authors of this study previously proposed an identification model of lookout activity using kinetic sensors [19]. However, there is still a lack of studies that investigate a navigator's behavior to measure maneuvering activity patterns in a ship collision environment.

The purpose of this study is to establish a navigator's behavior model for ship maneuvering using a ship maneuvering simulator in a collision-encountering situation. The proposed maneuvering behavior model employed 100 senior cadets at a maritime university in the Republic of Korea. In the given navigation scenarios, the participants performed maneuvering behavior to avoid collisions between ships. The maneuvering histories collected by the simulator system were utilized to develop two behavior models-i.e., the success and failure models for collision avoidance-which were validated in terms of their discrimination powers. The main contributions of this study are the following: a scientific research framework for the investigation of a navigator's error from his/her behavior for ship collision avoidance, and objective evidence of how a navigator maneuvers a ship in water using control devices.

\section{Materials and Methods}

The research framework of this study is presented in Figure 1. In the data processing phase (Phase 1), logged histories were obtained from ship maneuvering simulations. Then, maneuvering features were detected and sequence data were created to model maneuvering behavior. In the model development phase (Phase 2), maneuvering behavior models for the success and failure of collision avoidance were developed through model comparison.

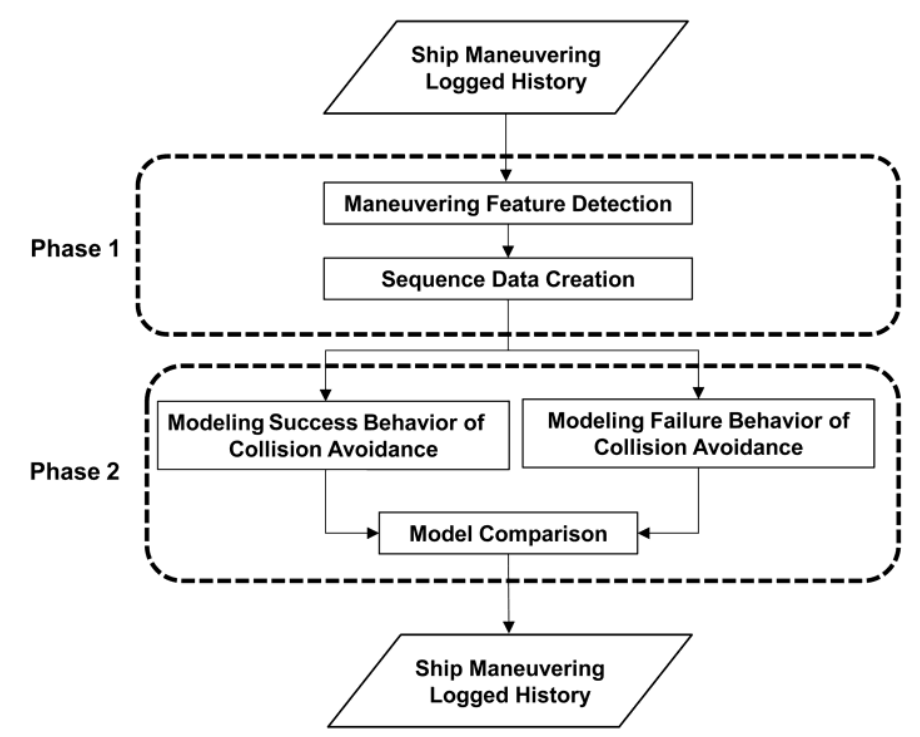

Figure 1. Research framework for the development of maneuvering behavior models for ship collision avoidance. 


\subsection{Data Collection}

\subsubsection{Experiment Configuration}

Logged histories were collected in the ship maneuvering simulator using the simulator system manufactured by Kongsberg digital AS (K-sim Polaris ship's S-bridge simulator) [20]. The Kongsberg simulator is a promising ship-handling training system, which uses maneuvering devices (i.e., steering wheel and engine telegraph), information display of navigation equipment (i.e., electronic chart, information system, and radar), and body-movement calculation software for a ship interacting with a user. Moreover, the Kongsberg simulator provides a human-machine interaction logging system between a user and a ship's body movement. The logged data can be acquired in the form of an Excel file containing ship maneuvering histories as a time series with two-second intervals. The experimental configuration with the Kongsberg simulator is illustrated in Figure 2. Figure 3 shows a simulation scene by a participant in the simulation scenarios, where collision avoidance action was required, as illustrated in Figure 4. In the simulation, two ships were used, i.e., a Tanker ship (length of $226 \mathrm{~m}$ ) as the own ship (OS) and a passenger ship (length of $260.8 \mathrm{~m}$ ) as the target ship (TS). Table 1 shows the specifications and initial conditions of ships used in the simulations.

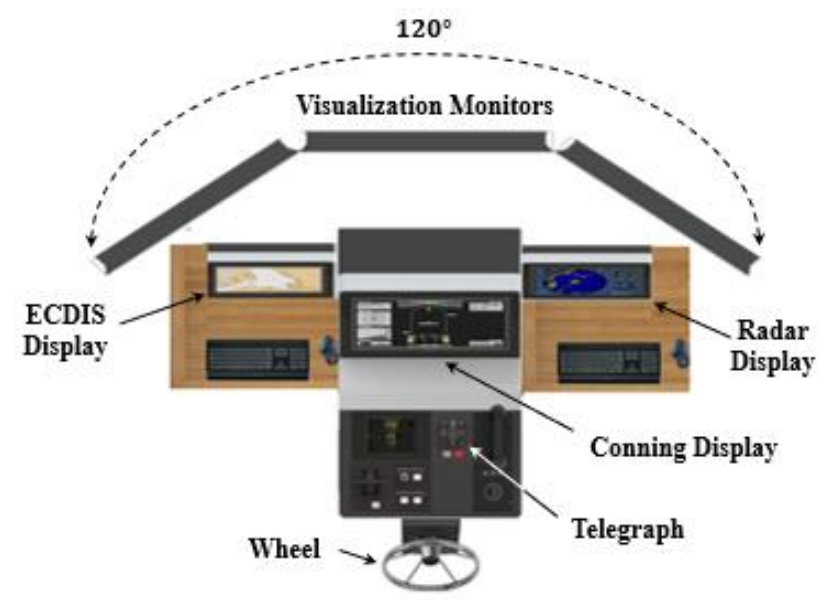

Figure 2. Experimental configuration with the Kongsberg ship-handling simulator.

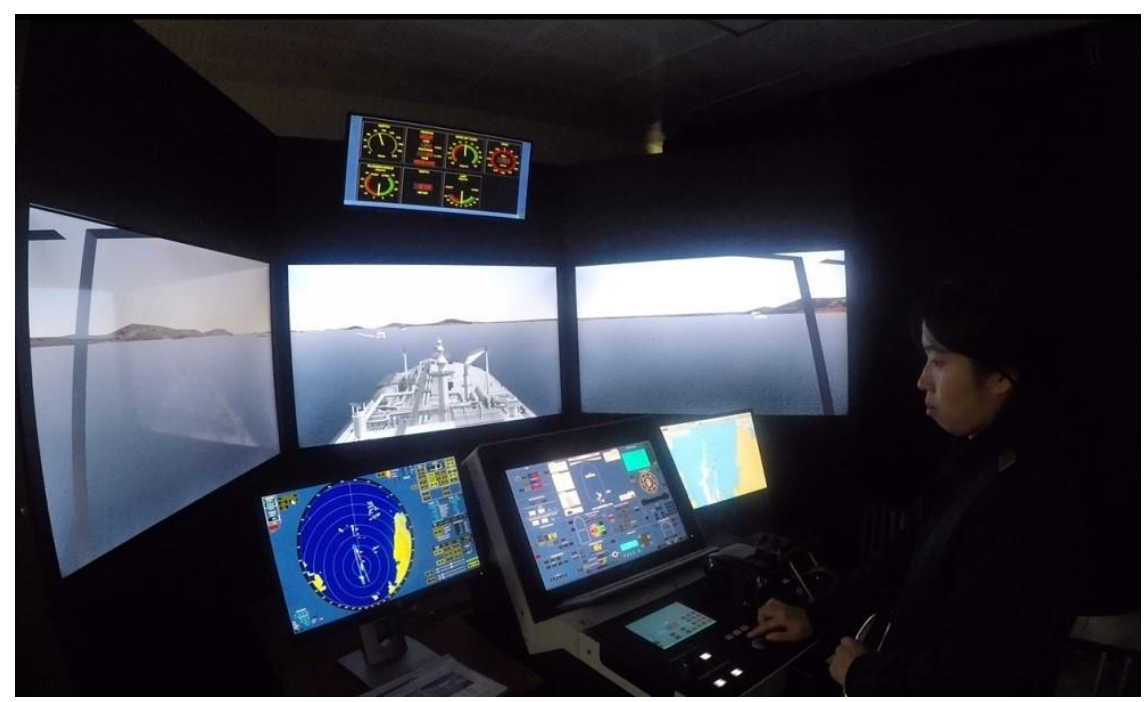

Figure 3. Snapshot of a scene in the simulation experiment. 


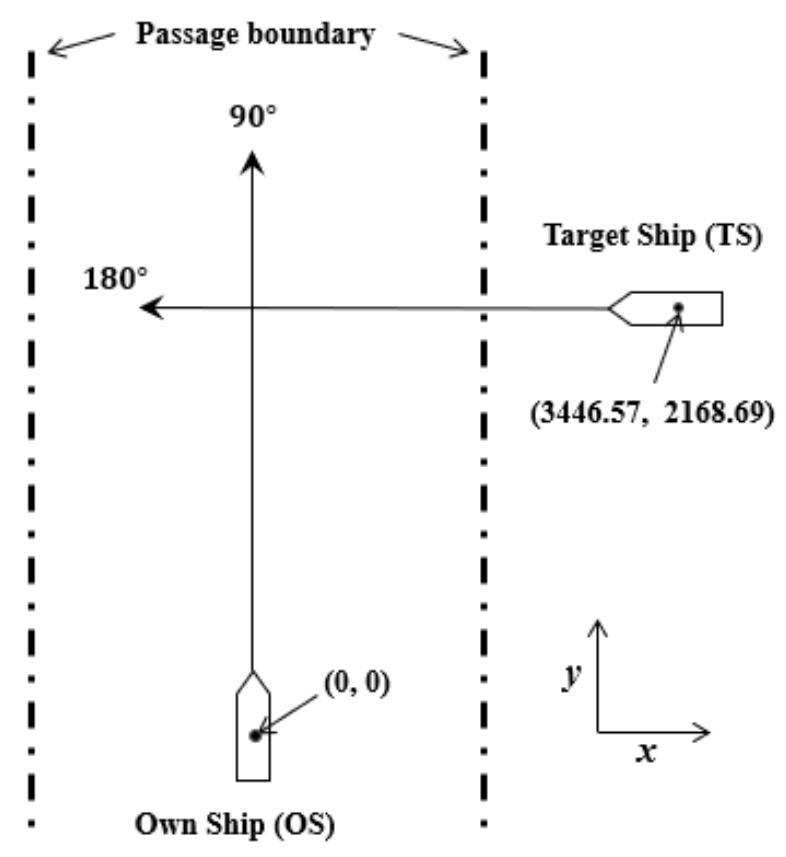

Figure 4. Simulation scenarios where collision avoidance action is required.

Table 1. Specifications and initial conditions of ships used in the simulations.

\begin{tabular}{cccc}
\hline \multirow{2}{*}{ Classification } & Factor & \multicolumn{2}{c}{ Ship Type } \\
\cline { 3 - 4 } & & $\begin{array}{c}\text { Tanker } \\
\text { (Own Ship) }\end{array}$ & $\begin{array}{c}\text { Cruise } \\
\text { (Target Ship) }\end{array}$ \\
\hline \multirow{2}{*}{ Specifications } & Length overall $(\mathrm{m})$ & 226.0 & 260.8 \\
& Breadth $(\mathrm{m})$ & 36.6 & 31.5 \\
& Maximum rudder angle $\left(^{\circ}\right)$ & \pm 45.0 & \pm 45.0 \\
& Maximum engine power & 13.507 & 8.441 \\
& Maximum speed $(\mathrm{m} / \mathrm{sec})$ & 8.7 & 11.8 \\
\hline \multirow{2}{*}{ Initial conditions } & Position $(x, y)$ & $(0,0)$ & $(3446.57,2168.69)$ \\
& Heading $\left({ }^{\circ}\right)$ & 90.0 & 180.0 \\
& Speed $(\mathrm{m} / \mathrm{sec})$ & 6.7 & 11.8 \\
\hline
\end{tabular}

\subsubsection{Experiment Protocol}

In the ship maneuvering simulations, 100 senior cadets at Mokpo National Maritime University participated in the study; their characteristics are shown in Table 2. These participants had taken maritime training courses for three and a half years at IMO-accredited maritime educational institutions, and have been in a merchant's vessel for more than a year. Also, all participants used the Kongsberg's simulator for at least $80 \mathrm{~h}$. To determine the appropriate sample size, the minimum sample size for the appropriate learning ability must be determined [21]. The dichotomous endpoint is a binary code: collision or not. The result was a minimum sample size of 122 persons $(\alpha \leq 0.05$, power $=0.8)$.

Table 2. Participant characteristics.

\begin{tabular}{cc}
\hline Characteristic & Mean (SD) \\
\hline$n$ & 100 \\
Female/male & $8 / 92$ \\
Age (year) & $22.6(1.2)$ \\
Onboard experience (year) & $0.9(0.2)$ \\
\hline
\end{tabular}


Table 3 shows the simulation procedure with the following three steps: $3 \mathrm{~min}$ of scenario explanation in step 1, $10 \mathrm{~min}$ of familiarity with the simulator in step 2, and about $5 \mathrm{~min}$ of simulation (ends when a collision occurs) in step 3. From the ship maneuvering simulation, the logged history data for the 100 participants were obtained; then the navigator's actions to avoid a collision were detected. To detect behavior actions, two types of behaviors, device and route control, were introduced. Device control refers to the use of rudder or engine. On the other hand, route control refers to the control of deviation or distance of the closest point of approaches (dCPA). The navigator's behavior was detected with four behavior features, i.e., rudder use, engine use, controlled deviation, and controlled dCPA. The types of behavior and their features are summarized in Table 4. Each behavior and its features assume that the navigator maneuvers the ship in accordance with international regulations for the prevention of collisions (COLREG) [22], as illustrated in Figure 5. Normally, ship maneuvering is implemented through the ship's heading and course, controlled by a rudder, as well as the ship's speed, controlled by an engine. When the navigator uses control devices (i.e., rudder and engine), there is a significant time delay between the ordered value and the response value of the devices. This time delay is a key characteristic caused by fluid pressure on the rudder and the hull [23]. Thus, ship maneuvering requires manipulative techniques of control devices. In order to avoid collisions along the designed route, the deviation between the vessel and the designed ship's route should be small, and the dCPA must be secured enough for collision avoidance. The closest point of approaches (CPA) represents an estimated point at which the distance between two objects (i.e., the OS and TS), where at least one is moving, reaches a minimum value. Thus, ship maneuvering for collision avoidance requires a technique to control the ship's route in addition to the device control technology. In this study, the maneuvering behavior model of the navigator was constructed using four behavior features mentioned above, and MATLAB 2008 (Mathworks, Natick, MA, United States) was used for the computations.

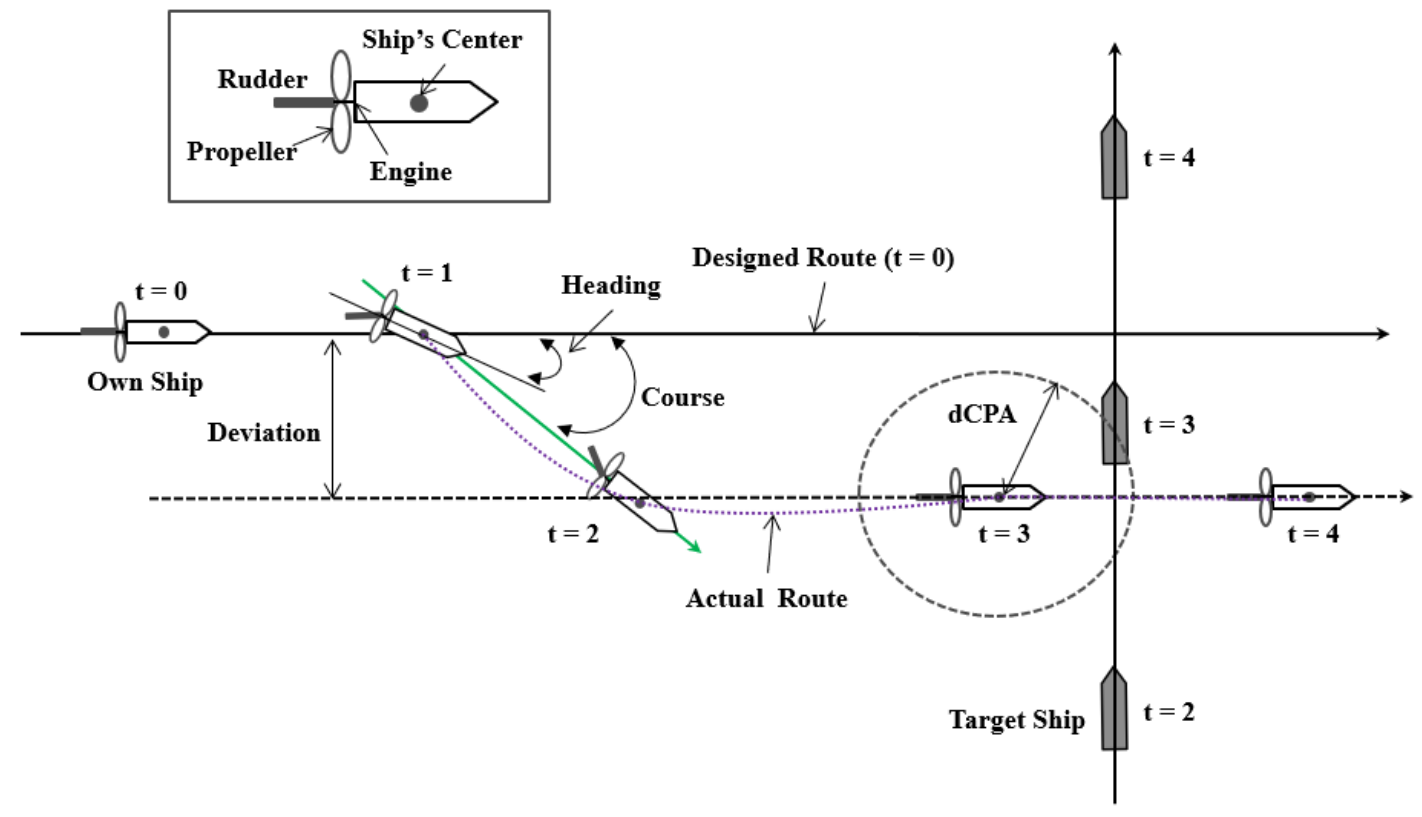

Figure 5. Example of a navigator's maneuvering behavior to avoid a collision.

Table 3. Simulation procedures.

\begin{tabular}{ccc}
\hline Procedure & Description & Duration (min) \\
\hline Step 1 & Explanation of scenario & 3 \\
Step 2 & $\begin{array}{c}\text { Familiarization of simulator } \\
\text { Simulation (ends when the own } \\
\text { ship collides with the target ship) }\end{array}$ & $\begin{array}{c}10 \\
\text { (variable) }\end{array}$ \\
\hline
\end{tabular}


Table 4. Types of behavior and behavior features used in the study.

\begin{tabular}{cc}
\hline Type of Behavior & Type of Behavior Feature \\
\hline Device Control & $\begin{array}{c}\text { Rudder use } \\
\text { Engine use }\end{array}$ \\
\hline Route Control & $\begin{array}{c}\text { Controlled deviation } \\
\end{array}$ \\
\hline
\end{tabular}

\subsection{Data Analysis}

\subsubsection{Data Processing}

Data processing consists of three tasks: converting the logged history into experimental data, detecting behavior features, and creating sequence data for behavior model development. For data conversion, the logged histories obtained from the maneuvering simulator were converted into experimental data, as described in Table 5. Ship maneuvering is represented by port and starboard movement and velocity changes of the ship. Here, the navigator uses the rudder for left and right movement, and the engine uses speed for movement [24,25]. Also, we selected four features because the risk of collision to both vessels can be assessed by deviation and dCPA [26]. For feature detection, using the detection rules presented in Table 6, four behavior features were detected with binary values ( 0 or 1 ). Two features, rudder use and engine use, were detected using a rudder reference value of $\pm 5^{\circ}$ and an engine reference value of $10.13 \mathrm{MW}$. The rudder reference value is the minimum effective angle required for ship control, and the engine reference value is $3 / 4$ of the maximum engine power [27]. In addition, the other two features, controlled deviation and controlled dCPA, were both detected using the same reference value of $361.6 \mathrm{~m}$. The reference value was calculated as the bumper area of OS, considering the width of the passage [28-30]. The bumper area was calculated as the room required for collision avoidance [31,32]. Note that unlike the detection of behavior features for device control, calculations of deviation and dCPA were required for the detection of behavioral features of route control.

Table 5. Types of experimental data obtained from the logged histories in the simulations.

\begin{tabular}{cc}
\hline Type & Description \\
\hline Time & Ship-handling time (seconds) \\
\hline Position & $\begin{array}{c}\text { Ship's position }(x, y) \text { : converted from position } \\
\text { (latitude, longitude) in the geographic coordination } \\
\text { system (ECS) to Cartesian coordinate system }\end{array}$ \\
\hline Course & $\begin{array}{c}\text { Direction of ship's route }\left(^{\circ}\right) \text { : converted from the } \\
\text { azimuth in ECS to Cartesian coordinate system }\end{array}$ \\
\hline Speed & $\begin{array}{c}\text { Ship's speed }(\mathrm{m} / \mathrm{sec}) \text { : Converted from knots } \\
\text { (mile/hour) to speed }(\mathrm{m} / \mathrm{sec})\end{array}$ \\
\hline Engine power & Used engine power $(\mathrm{MW})$ \\
\hline Rudder angle & Ordered rudder angle $\left(^{\circ}\right)$ \\
\hline
\end{tabular}


Table 6. Rules for the detection of four maneuvering behavior features.

\begin{tabular}{|c|c|c|c|c|}
\hline Behavior & Feature & Rule & Value & Description \\
\hline \multirow{2}{*}{ Device Control } & Rudder Use & $\begin{array}{l}o R A(t)<R A_{r e f} \\
o R A(t) \geq R A_{r e f}\end{array}$ & $\begin{array}{l}0 \\
1\end{array}$ & $\begin{array}{c}o R A(t): \text { Ordered rudder angle at time } t \\
R A_{\text {ref }}: \text { Reference angle of } \pm 5^{\circ}\end{array}$ \\
\hline & Engine Use & $\begin{array}{l}o E P(t)<E P_{\text {ref }} \\
o E P(t) \geq E P_{\text {ref }}\end{array}$ & $\begin{array}{l}0 \\
1\end{array}$ & $\begin{array}{l}o E P(t): \text { Used engine power at time } t \\
E P_{\text {ref }}: \text { Reference power of } 10.13 \mathrm{MW}\end{array}$ \\
\hline \multirow{2}{*}{ Route Control } & $\begin{array}{l}\text { Controlled } \\
\text { Deviation }\end{array}$ & $\begin{array}{l}\operatorname{Dev}(t)<D e v_{r e f} \\
\operatorname{Dev}(t) \geq \operatorname{Dev} r e f\end{array}$ & $\begin{array}{l}0 \\
1\end{array}$ & $\begin{array}{c}\operatorname{Dev}(t): \text { Deviation }{ }^{1} \text { at time } t \\
\operatorname{Dev}_{r e f}: \text { Reference distance of } 361.6 \mathrm{~m}\end{array}$ \\
\hline & Controlled dCPA & $\begin{array}{l}d C P A(t)<d C P A_{r e f} \\
\mathrm{~d} C P A(t) \geq d C P A_{r e f}\end{array}$ & $\begin{array}{l}0 \\
1\end{array}$ & $\begin{array}{c}d C P A(t): \mathrm{dCPA}^{2} \text { at time } t \\
d C P A_{\text {ref }}: \text { Reference distance of } 361.6 \mathrm{~m}\end{array}$ \\
\hline
\end{tabular}

${ }^{1}$ Calculated deviation using Equation (1); ${ }^{2}$ Calculated distance of the closest point of approaches (dCPA) using Equation (2).

The deviation $\operatorname{Dev}(t)$ at time $t$ between the position of the OS and the designed route at the beginning of the simulation was calculated as

$$
\operatorname{Dev}(t)=\sqrt{\left(\psi_{O S(x)}(t)-\psi_{P R(x)}(t)\right)^{2}+\left(\psi_{O S(y)}(t)-\psi_{P R(y)}(t)\right)^{2}},
$$

where $\psi_{O S(x, y)}$ is the coordinate set of the position $\left(x_{O S}, y_{O S}\right)$ of the OS at time $t ; \psi_{P R(x, y)}=\left(x_{P R}, y_{P R}\right)$ is the coordinate set of the estimated position of the designed route, which was calculated with the course and speed of the OS at time $t=0$.

The calculation of the distance of the CPA requires complex procedures and formulas, which have already been reported in various references [33,34]. Using the formulas in [35], $d C P A(t)$ was calculated using the following procedure:

- $\quad$ Step 1: Let $\{\psi, v, \theta\}$ be the set of position $(\psi)$, speed $(v)$, and course $(\theta)$ at time $t$.

- Step 2: Obtain the set $\left\{\psi_{O S}, v_{O S}, \theta_{O S}\right\}$ of the OS and the set $\left\{\psi_{T S}, v_{T S}, \theta_{T S}\right\}$ of the TS.

- Step 3: Estimate the position of intersection points $\psi_{\text {Inter }}$ between $\theta_{O S}$ and $\theta_{T S}$ using the "navfix" program in MATLAB 2008 (Mathworks, Natick, MA, United States). Then, let $\hat{\psi}_{\text {OS }}$ be the position of OS at $\psi_{C S P}$.

- $\quad$ Step 4: Calculate the travel time $T T_{O S}$ of the OS from $\psi_{O S}$ to $\hat{\psi}_{O S}$ using $v_{O S}$.

- Step 5: Calculate the position $\hat{\psi}_{T S}$ of the TS at TTOS using $\theta_{T S}$.

- Step 6: Calculate $d C P A(t)$ between $\hat{\psi}_{O S}$ and $\hat{\psi}_{T S}$ at time $t$.

- Step 7: Iterate Steps 1 to 6 to $t=T$ ( $T$ is simulation time) and finish.

For sequence data creation, the four behavior features mentioned above were used. The performance levels of the device control $P L_{D O B}$ and the route control $P L_{R C B}$ were calculated as

$$
\begin{aligned}
& P L_{D O B}(n)=\frac{1}{T} \sum_{t=0}^{T}\left(D O B_{R u d}(n, t)+D O B_{E n g}(n, t)\right), \\
& P L_{R C B}(n)=\frac{1}{T} \sum_{t=0}^{T}\left(R C B_{D e v}(n, t)+R C B_{d C P A}(n, t)\right),
\end{aligned}
$$

respectively, where $n$ is the participant number, with $n=1,2,3, \cdots N(N=100) ; t$ is the simulation time that varies for each participant; $D C B_{R u d}$ is the detected binary value of the device control behavior for rudder use; $D C B_{\text {Eng }}$ is the detected binary value of the device control behavior for engine usage; $R C B_{D e v}$ is the detected binary value of the route control behavior with respect to the controlled deviation; and $R C B_{d C P A}$ is the detected binary value of the route control behavior for the controlled dCPA. 
In the above Equations (2) and (3), all four variables were applied during the simulation time $t$. However, interactions between the four variables and the maximum value of simulation time $t$ are raised as issues, and will be discussed in Section 4.

The performance level calculated by Equations (2) or (3) has a value between 0 and 1 . A value of 1 means that the navigator's device control and route control techniques are at a higher level compared to zero. Using the calculated performance levels for 100 participants, behavior sequences with symbols $v=\{$ Poor, Average, Excellent $\}$ were created, using the rules of symbolic performance levels in Table 7 . Then, the sequence data were constructed by allocating the success $O_{\text {Success }}$ and the failure $O_{\text {Fail }}$ of collision avoidance into the following $2 \times N$ ( $N$ is the sequence length) matrices:

$$
O_{\text {Success }}=\left[\begin{array}{c}
O_{D C B \mid \text { Success }} \\
O_{R C B \mid \text { Success }}
\end{array}\right] \text { and } O_{\text {Fail }}=\left[\begin{array}{c}
O_{D C B \mid F a i l} \\
O_{R C B \mid F a i l}
\end{array}\right] \text {, }
$$

where $O_{(D C B \mid S u c c e s s)} \in v$ and $O_{R C B \mid \text { Success }} \in v$ are the device control behavior and the route control behavior for the success of collision avoidance, respectively, while $O_{D C B \mid F a i l} \in v$ and $O_{R C B \mid F a i l} \in v$ are the device control behavior and the route control behavior for the failure of collision avoidance, respectively.

Table 7. Rules for symbolic performance levels.

\begin{tabular}{ccc}
\hline Performance Level & Symbol & Rule \\
\hline Low & Poor & $P L_{*}^{3}=1 / 3$ \\
Moderate & Average & $1 / 3<=P L_{*}<2 / 3$ \\
High & Excellent & $P L_{*}>=2 / 3$ \\
${ }^{3}$ Behavior performance level of the device control $\left(P L_{D O B}\right)$ or of the route control $\left(P L_{R C B}\right)$.
\end{tabular}

\subsubsection{Model Development}

During the model development phase, the rules of the Markov chain and its related methods were used [36-43]. The developed behavior model $\lambda(A, B, \pi)$ with two states and three parameters $\mathrm{A}, \mathrm{B}$, and $\pi$ is as follows:

- State transition probability distribution: $A=\left\{a_{i j}\right\}=\left[\begin{array}{ll}a_{11} & a_{12} \\ a_{21} & a_{22}\end{array}\right]$

- Observation symbol probability distribution: $B=\left\{b_{j}\left(v_{k}\right)\right\}=\left[\begin{array}{lll}b_{1}\left(v_{1}\right) & b_{1}\left(v_{2}\right) & b_{1}\left(v_{3}\right) \\ b_{2}\left(v_{1}\right) & b_{2}\left(v_{2}\right) & b_{2}\left(v_{2}\right)\end{array}\right]$

- Initial state distribution: $\pi=\left\{\pi_{i}\right\}=\left[\begin{array}{ll}1 & 1 \\ 1 & 1\end{array}\right]$

Adopting the classic model notation for a hidden Markov model (HMM) from Rabiner and Juang [36], the HMM is characterized by five elements, namely $N, M, A, B$, and $\pi$. These are described as follows. $N$ is the number of states in the model. The individual states and the state at time $t$ are denoted as $S=\left\{S_{1}, S_{2}, \cdots, S_{N}\right\}$ and $q_{t}$, respectively. $M$ is the number of observation symbols. The individual observation symbols are denoted as $V=\left\{v_{1}, v_{2}, \cdots, v_{M}\right\}$. $A$ is the state transition probability distribution, $A=\left\{a_{i j}\right\}$, where $a_{i j}=P\left[q_{t+1}=S_{j} \mid q_{t}=S_{i}\right], 1 \leq i, j \leq N$. The term $a_{i j}$ represents the transition probability from state $S_{i}$ to state $S_{j}$, and is subject to the constrains of $\sum_{j}^{N} a_{i j}=1$ and $a_{i j} \geq 0 . B$ is the observation symbol probability distribution in state $j: B=\left\{b_{j}(k)\right\}$, where $b_{j}(k)=P\left[v_{k}\right.$ at $\left.t \mid q_{t}=S_{j}\right]$, $1 \leq j \leq N$, and $1 \leq k \leq M$. The term $b_{j}(k)$ represents the probability of state $j$ at time $t$ with observation symbol $v_{k}$, and is subject to the constraints of $\sum_{k}^{M} b_{j}(k)=1$ and $b_{j}(k) \geq 0$. Finally, $\pi$ is the initial state probability distribution $\pi=\left\{\pi_{i}\right\}$, where $\pi_{i}=P\left[q_{1}=S_{i}\right], 1 \leq i \leq N$. The term $\pi_{i}$ represents the probability of $S_{i}$ being the initial state at time $t=1$.

Given appropriate values of $N, M, A, B$, and $\pi$, the HMM can be used to generate an observation sequence $O=O_{1} O_{2} \cdots O_{T}$ in which each observation $t$ is a symbol from $V$, and $T$ is the number of 
observations in the sequence. Given a finite observation sequence of hidden states, all the possible transition probabilities and symbol output probabilities can be multiplied at each transition to calculate the overall likelihood of all the output symbols produced in the transition path up to that point. Summing all such transition paths, one can then compute the likelihood of the HMM generating the sequence. The compact notion $\lambda=(A, B, \pi)$ can be used to represent the complete parameter set of the model. With HMMs, there are three basic problems that must be addressed, namely (i) evaluation, (ii) decoding, and (iii) learning.

The first problem is evaluation, i.e., the probability $P(\mid \lambda)$ of the model $\lambda=(A, B, \pi)$ producing a given sequence $O=O_{1} O_{2} \cdots O_{T}$. The evaluation problem can be solved using the forward-backward dynamic programming algorithm $[37,38]$. The forward variable $\alpha_{t}(j)$ is defined as the probability of the partial observation sequence $O_{1} O_{2} \cdots O_{t}$ and state $S_{i}$ at time $t$, given the model $\lambda$. The variable $\alpha_{t}(j)$ can be calculated recursively by (i) initializing $\alpha_{1}(i)$, as in Equation (4); (ii) calculating $\alpha_{t+1}(j)$, as in Equation (5); and (iii) calculating $P(\mid \lambda)$, as in Equation (6):

$$
\begin{gathered}
\alpha_{1}(i)=\pi_{i} b_{i}\left({ }_{1}\right), 1 \leq i \leq N, \\
\alpha_{t+1}(j)=b_{j}(t+1) \sum_{i=1}^{N} \alpha_{t}(i) a_{i j}, 1 \leq t \leq T-1,1 \leq j \leq N, \\
P(\mid \lambda)=\sum_{i=1}^{N} \alpha_{T}(i)
\end{gathered}
$$

In addition, the backward variable $\beta_{t}(i)$ is defined as the probability of the partial observation sequence from $t+1$ to the final given state $S_{i}$ at time $t$, as well as the model $\lambda$. The variable $\beta_{t}(i)$ is calculated recursively by (i) initializing $\beta_{T}(i)$, as in Equation (7); and (ii) calculating $\beta_{t}(i)$, as in Equation (8):

$$
\begin{gathered}
\beta_{T}(i)=1,1 \leq i \leq N \\
\beta_{t}(i)=\sum_{j=1}^{N} \beta_{t+1}(j) a_{i j} b_{j}(t+1), t=T-1, T-2, \cdots, 1,1 \leq i \leq N
\end{gathered}
$$

The second problem is decoding, i.e., determining the most probable path of hidden states given the sequence of observations. A common means of solving this problem is the Viterbi algorithm [39,40], which uses a forward-backward algorithm across the trellis of hidden states to find a single best-state sequence $Q=\left\{q_{1} q_{2} \cdots q_{T}\right\}$ for a given observation sequence $O=\left\{O_{1} O_{2} \cdots O_{T}\right\}$. To do so, the highest probability $\delta_{t}(i)$ along a single path at time $t$ must be defined, which accounts for the first $t$ observations and ends in state $S_{i}$. The probability $\delta_{t}(i)$ is defined as in Equation (9), and is calculated recursively, as in Equation (10):

$$
\begin{gathered}
\delta_{t}(i)=\max _{q_{1}, q_{2}, \cdots, q_{t-1}} P\left[q_{1} q_{2} \cdots q_{t}=i, O_{1} O_{2} \cdots O_{t} \mid \lambda\right], \\
\delta_{t+1}(j)=\left[\max _{i} \delta_{t}(i) a_{i j}\right] b_{j}(t+1) .
\end{gathered}
$$

The third problem is model learning, i.e., adjusting the model parameters $\lambda=(A, B, \pi)$ to maximize the probability $P(\mid \lambda)$ given the observation sequence. Several methods can be used to estimate the model parameters, the main ones being supervised learning algorithms and unsupervised learning algorithms. A supervised learning algorithm is a maximum likelihood estimation method [41,42] that uses observation sequences and the corresponding state sequence data, while the unsupervised learning algorithm is a forward-backward algorithm that uses observation sequences. In the present study, the model parameters of participant behavior are obtained using supervised learning.

Figure 6 shows a graphical representation of the two-state ergodic hidden Markov model (HMM) used in the behavior model development. Two types of behavior models were developed in this 
study, the success model $\lambda_{\text {Sucess }}$ and the failure model $\lambda_{\text {Fail }}$, which were trained by the sequence data observed from the success and the failure of collision avoidance, respectively. The developed behavior models were validated using model distance and model performance. Model distance evaluates the dissimilarity between two models [43], while model performance measures the correct answering percentile (\%) for observations generated by the model. The model distances $\left(M D_{\text {Success }}\right.$ or $\left.M D_{\text {Fail }}\right)$ between $\lambda_{\text {Sucess }}$ and $\lambda_{\text {Fail }}$ were calculated as

$$
\begin{gathered}
M D_{\text {Success }}=M D\left(\lambda_{\text {Fail }}, \lambda_{\text {Success }}\right)=\frac{1}{T}\left[\log P\left(O_{\text {Success }} \mid \lambda_{\text {Fail }}\right)-\log P\left(O_{\text {Success }} \mid \lambda_{\text {Success }}\right)\right], \\
M D_{\text {Fail }}=M D\left(\lambda_{\text {Success }}, \lambda_{\text {Fail }}\right)=\frac{1}{T}\left[\log P\left(O_{\text {Fail }} \mid \lambda_{\text {Success }}\right)-\log P\left(O_{\text {Fail }} \mid \lambda_{\text {Fail }}\right)\right]
\end{gathered}
$$

where $O_{\text {Success }}$ is the sequence of observations generated by model $\lambda_{\text {Success, }}$ and $O_{\text {Fail }}$ is the sequence of observations generated by model $\lambda_{\text {Fail }}$.

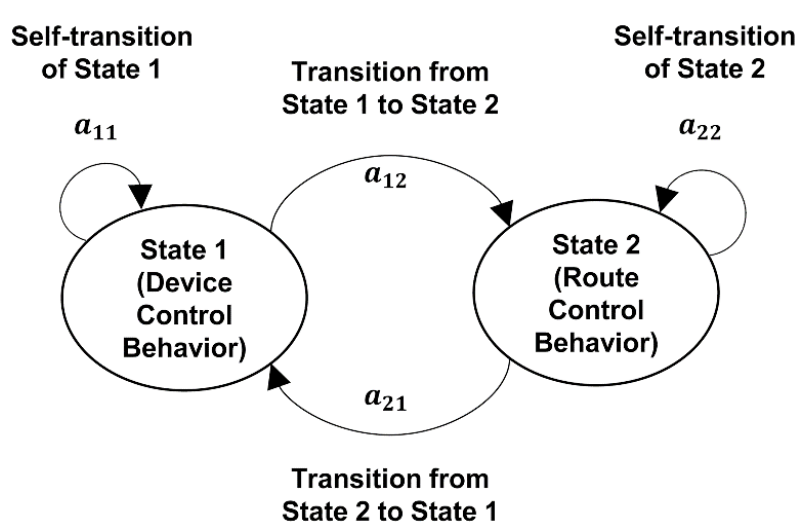

Figure 6. Graphical representation of the behavior model with a two-state ergodic hidden Markov model (HMM).

The model performances $\left(C R_{\text {Success }}\right.$ or $\left.C R_{\text {Fail }}\right)$ for $M D_{\text {Success }}$ or $M D_{\text {Fail }}$ were calculated as

$$
\begin{gathered}
C R_{\text {Success }}=\sum_{t=1}^{T} I,\left(I=1, \text { if } M D_{\text {Success }}>0\right), \\
C R_{\text {Fail }}=\sum_{t=1}^{T} I,\left(I=1, \text { if } M D_{\text {Fail }}>0\right)
\end{gathered}
$$

\section{Results}

\subsection{Feature Detection}

Figure 7 shows an example of behavior feature detection of a participant who failed to avoid a collision. It can be seen that the participant used both the rudder and the engine for a certain period, but the deviation and the dCPA fluctuated around their reference values (dotted lines) for $5 \mathrm{~min}$. The feature detections for all participants $(n=100)$ are shown in Figure 8, which were observed from 47 who were successful in collision avoidance and 53 who failed to avoid collision. Figure 9 shows the percentile (\%) of behavior performance levels divided into two types: successful collision avoidance and failed collision avoidance. Analysis of the "excellent" levels in these two types showed that Figure $9 \mathrm{a}$,c show $51.66 \%$ and $72.34 \%$, respectively, for successful collision avoidance, while Figure $9 \mathrm{~b}, \mathrm{~d}$ show $5.66 \%$ and $3.77 \%$, respectively. From the results of Figure 9, it was found that the percentile of "excellent" levels in the successful behavior was about 10 to 20 times higher than that of the unsuccessful behavior. Thus, the sequence data constructed from the behavior feature detection have a 
behavior feature that can clearly distinguish between success and failure of collision avoidance. Using the sequence data shown in Figure 8, two behavior models divided into the success and failure of collision avoidance were constructed.
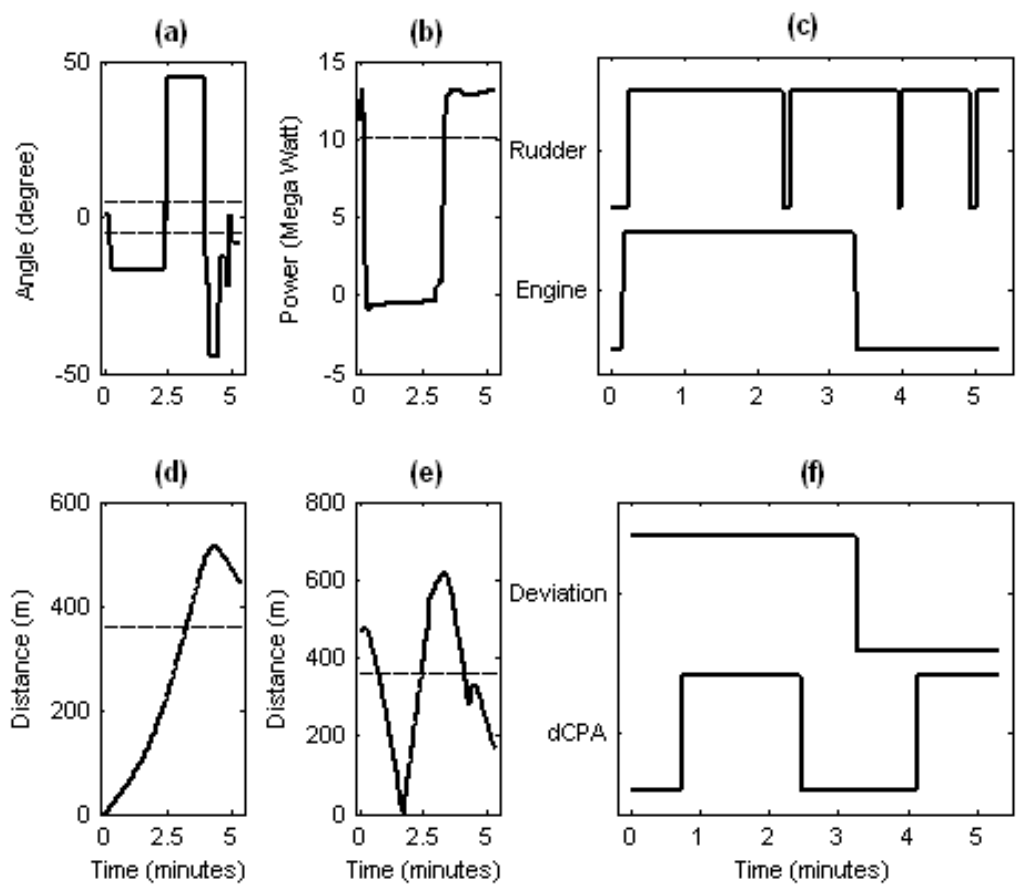

Figure 7. Examples of behavior feature detection of a participant who failed to avoid a collision: (a) rudder angle; (b) engine power; (c) feature detection results for $(\mathbf{a}, \mathbf{b})$; (d) controlled deviation; (e) controlled dCPA; and (f) feature detection results for $(\mathbf{d}, \mathbf{e})$.

(a)

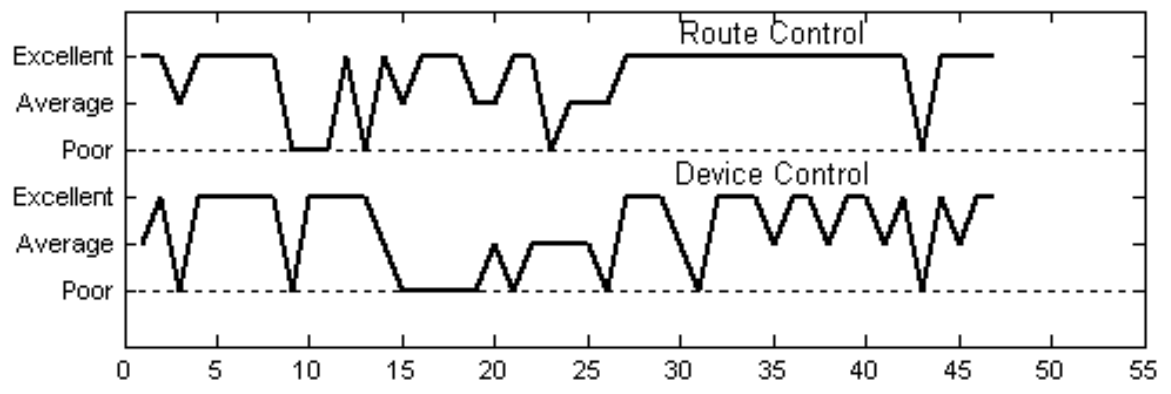

(b)

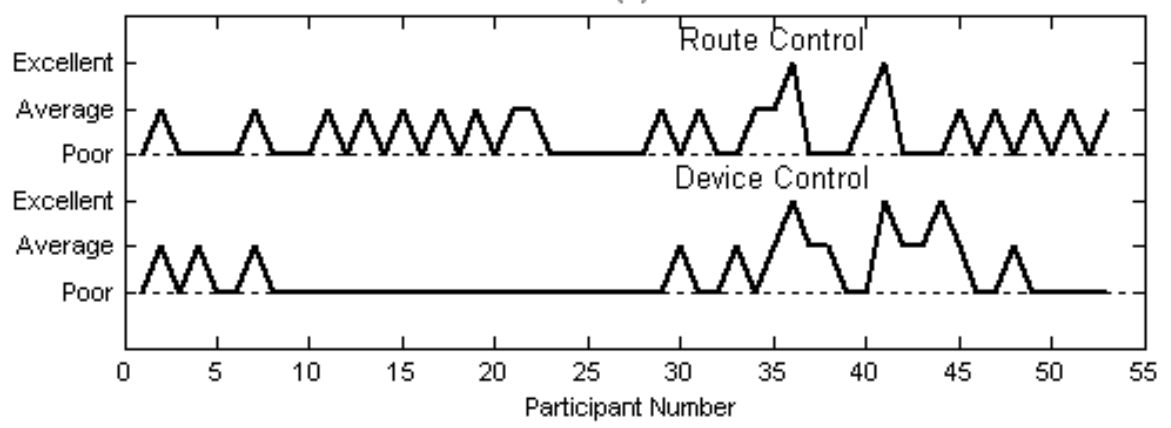

Figure 8. Observed sequence data for (a) device control behavior and route control behavior for the success of collision avoidance, and (b) device control behavior and route control behavior for the failure of collision avoidance. 
(a)

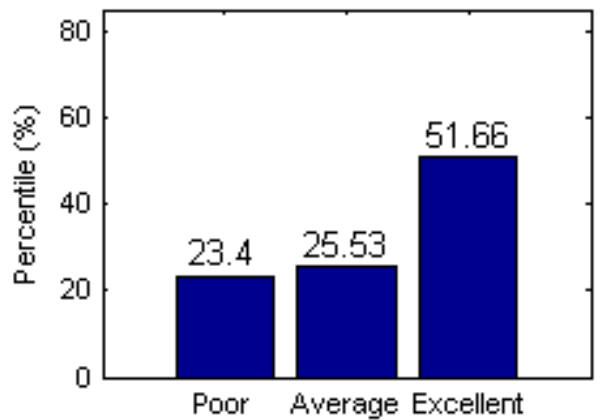

(c)

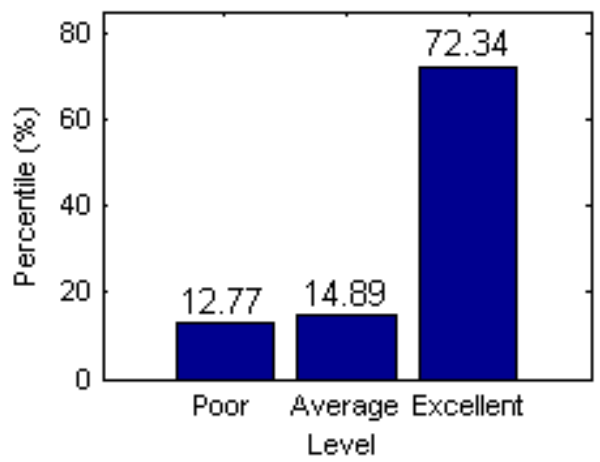

(b)

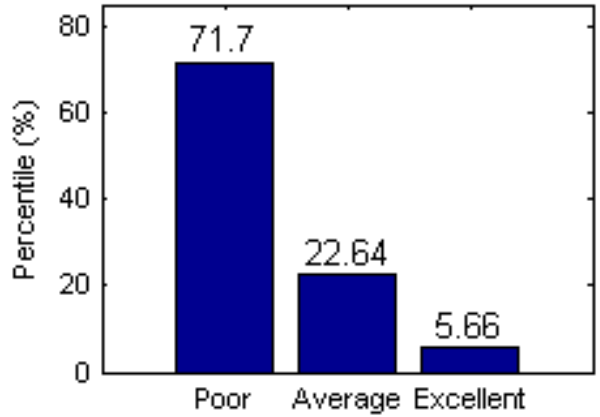

(d)

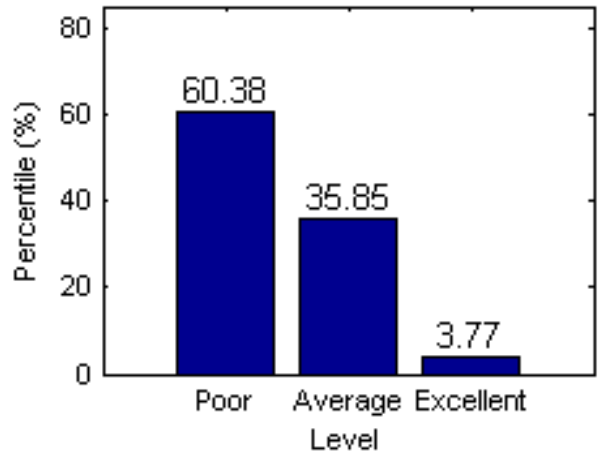

Figure 9. Percentile (\%) of behavior performance levels for successful and failed collision avoidance; (a) device control behavior for success; (b) device control behavior for failure; (c) route control behavior for success; and (d) route control behavior for failure.

\subsection{Model Validation}

The developed behavior models were validated in terms of model distance and model performance. Table 8 provides answers to the following questions: given two HMMs, the failure model $\lambda_{\text {Fail }}$ and the success model $\lambda_{\text {Success }}$, what is a reasonable measure of the similarity of the two models? An important key point here is similarity criteria. Even though the two models, $\lambda_{\text {Fail }}$ and $\lambda_{\text {Success }}$, look very different, a statistical equivalent of the model can occur. Distance measures $D\left(\lambda_{\text {Fail }}, \lambda_{\text {Success }}\right)$ or $D\left(\lambda_{\text {Success }}, \lambda_{\text {Fail }}\right)$ between models $\lambda_{\text {Fail }}$ and $\lambda_{\text {Success }}$ have a dissimilarity concept with respect to model distance. Several interpretations of the model distance exist in terms of such as cross entropy, divergence, and discrimination of information $[28,35]$. The $M D_{\text {Success }}$ is a measure result of how well the model $\lambda_{\text {Fail }}$ matches observations generated by model $\lambda_{\text {Success, }}$, relative to how well model $\lambda_{\text {Success }}$ matches observations generated by itself. Also, $M D_{\text {Fail }}$ is a measure result of how well model $\lambda_{\text {Success }}$ matches observations generated by model $\lambda_{\text {Fail }}$, relative to how well model $\lambda_{\text {Fail }}$ matches observations generated by itself.

Table 8. Comparison results of two models in terms of model distance and model performance.

\begin{tabular}{ccc}
\hline Classification & Measurement Variable & Mean (SD) \\
\hline \multirow{2}{*}{ Model distance } & $M D_{\text {Success }}=D\left(\lambda_{\text {Fail }}, \lambda_{\text {Success }}\right)$ & $-2.60(2.68)$ \\
& $M D_{\text {Fail }}=D\left(\lambda_{\text {Success }}, \lambda_{\text {Fail }}\right)$ & $-1.69(1.54)$ \\
\hline \multirow{2}{*}{ Model performance } & $C R_{\text {Success }}$ & 0.7871 \\
& $C R_{\text {Fail }}$ & 0.9434 \\
\hline
\end{tabular}

Table 8 shows the validation results by comparing the two models. Note that a larger model distance indicates greater discernibility of the model. Meanwhile, model performance increases as the model distance increases. The $\log$ likelihood (-1.69) of $M D_{\text {Success }}$ was about 1.5 times larger than 
the log likelihood (-2.60) of $M D_{\text {Fail }}$. Thus, dissimilarity was greater for the failure model than that for the success model. As a result, the failure model shows that the degree of dissimilarity with the success model is larger than the success model, but more closely matches the observations generated by the model itself. This result implies that the failure model has a higher predictive power of behavior observed compared to the success model. The reason why the failure model has higher discriminative power than the success model will be discussed in Section 4 .

As the result of model distance, the percentile of model performance was $78.71 \%$ for the success model and $94.34 \%$ for the failure model.

\subsection{Behavior Analysis}

The maneuvering behavior of a navigator was analyzed using two behavior models, i.e., a success model of collision avoidance and a failure model of collision avoidance. Figure 10 shows the transition probability of two states for both models. The analysis results of both models are summarized as follows.

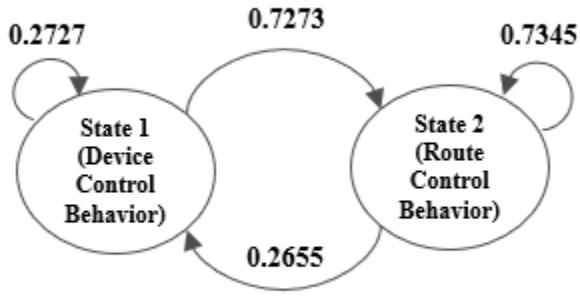

(a)

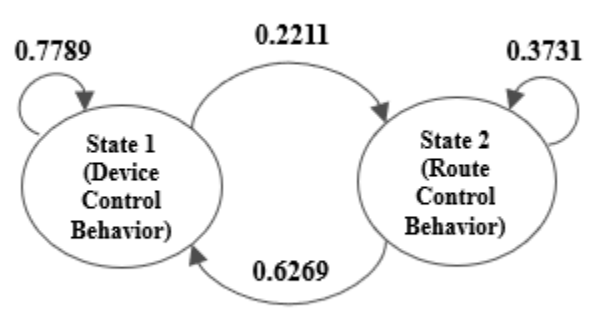

(b)

Figure 10. Maneuvering behavior models: (a) success of collision avoidance, and (b) failure of collision avoidance.

First, for the success model, the probability (0.7273) of transition from state 1 to state 2 was about 2.7 times larger than that from state 2 to state 1 (probability of 2.655). On the other hand, for the failure model, the probability (0.6269) of transition from state 2 to state 1 was about 2.8 times larger than that from state 1 to state 2 (probability 0.2211). As results, we found that the two key features; (i) navigators who succeeded in collision avoidance showed that the device control behavior was predominantly transitioned to route control behavior, and (ii) navigators who failed in collision avoidance showed that the route control behavior was predominantly transitioned to device control behavior.

Second, for the success model, the self-transition probability (0.7345) of state 2 is about 2.7 times larger than that of state 1 (probability of 0.2727 ). On the other hand, for the failure model, the self-transition probability (0.7789) of the state 1 is about 2.1 times larger than that of state 2 (probability of 0.3731 ). From these results, we found that (i) navigators who succeeded in collision avoidance showed a self-transition predominance of the route control behavior, and (ii) navigators who failed to avoid the collision showed a self-transition predominance of the device control behavior.

In the above results, the behavior of the navigators was clearly identified for both successful and unsuccessful collisions. The reason why the success and failure models have different transition characteristics will be discussed in Section 4.

\section{Discussion}

This study was conducted for developing a collision encountering situation in a simulator using a navigator's behavior model for ship maneuvering. The main contribution of this study is the development of success and failure models to investigate navigator errors from behavior on ship collision avoidance. As mentioned in Section 2.2.1, four variables-rudder, engine, planned route control, and dCPA control-have been applied for the performance level calculation during the simulation time. The reason why these four variables were applied during the simulation time can 
be explained as follows. First, planned route tracking and dCPA control require both rudder and engine use. Second, changing the vessel course for planned route tracking can lead to a change in dCPA. Third, changing the ship course for dCPA control can cause changes in planned route tracking. As in these three explanations, the four variables have mutual influences. Thus, in this study, the four variables are used in the performance level calculation during simulation time. Moreover, as mentioned in Section 2.2.1, the maximum time estimate for calculating performance levels has been an issue. Unfortunately, the maximum time required to calculate performance levels is not known [36]. The reason for this is that depending on the given situation, the possibility of a performance level varies. Thus, in this study, a collision-prone scenario is applied to the simulation experiment. A study on the estimation of the optimal time required to calculate the performance level was left as a future research work.

As mentioned in Section 2.2.2, the failure model had higher discriminative power than the success model. This can be interpreted as follows. Since collision avoidance failure was determined after a normal navigational situation, both models had normal navigation situations [44-46]. In particular, the success model reflects more of the normal navigation situation compared to the failure model. Thus, the failure model can be interpreted as having greater discriminative power than the success model. On the other hand, as mentioned in Section 3.3, different transition characteristics were found between the success and failure models. This can be interpreted as follows. In ship maneuvering, device control technology and route control techniques have complementary characteristics $[47,48]$. Successful route control requires a high level of device control technique. Also, the device control effect can be confirmed in a short amount of time compared to the route control effect, and effective route control requires long boarding experience and knowledge. Hence, this results in differences in transfer characteristics for the models.

It is important to distinguish between measurements in this real-field environment and measurements in virtual reality. The real-field environment was practically difficult due to time and cost [24]. Because this is the reason for the use of virtual reality, the results are expected to be real-field [49]. For the same reason, it is necessary to perform additional verification, because it is an experiment in which the participants of the experiment are aimed at the reserve navigator. Also, the rudder and engine have been used for the analysis of ship's course-keeping control in maritime fields [50], but we propose an analysis method for the error of the navigator divided by device control behavior and route control behavior.

This problem in ship control techniques has been raised previously in ship navigation, and it is about whether the technique comes first or experience [51,52]. This will be the subject of further research.

\section{Conclusions}

The results of the proposed maneuvering behavior models showed that the behavior of navigators was clearly identified for both the success and failure of collision avoidance. The proposed behavior models provide a better understanding of how a navigator behaves during collision-encountering situations, which can be applied to research of human error patterns for the prevention of navigational accidents, by showing harmful behavior patterns of the navigator. Future work will examine the association between a navigator's behavior patterns and maritime accidents by precisely anticipating possible navigator errors, in order to prevent maritime accidents.

Author Contributions: Conceptualization, J.-B.Y.; methodology, J.-B.Y.; software, I.-H.Y.; validation, D.-J.P. and I.-H.Y.; data curation, D.-J.P.; writing — original draft preparation, J.-B.Y.; writing-review and editing, D.-J.P. and I.-H.Y.; supervision and funding acquisition, J.-B.Y.

Funding: The contents of this paper are the results of the research project of the Ministry of Oceans and Fisheries of Korea (fundamental research on maritime accident prevention-phase 2).

Conflicts of Interest: The authors declare no conflict of interest. 


\section{References}

1. Corporate, A.G. Safety and Shipping Review; Allianz Global Corporate and Specialty: Hong Kong, China, 2015.

2. Corporate, A.G. Safety and Shipping Review; Allianz Global Corporate and Specialty: Hong Kong, China, 2016.

3. Corporate, A.G. Safety and Shipping Review; Allianz Global Corporate and Specialty: Hong Kong, China, 2017.

4. Boussemart, Y.; Cummings, M.L. Behavioral recognition and prediction of an operator supervising multiple heterogeneous unmanned vehicles. Hum. Oper. Unmanned Syst. 2008. Available online: https://s3.amazonaws. com/academia.edu.documents/3240124/12-boussemart08humous.pdf (accessed on 27 May 2019).

5. Boussemart, Y.; Las Fargeas, J.; Cummings, M.L.; Roy, N. Comparing learning techniques for hidden Markov Models of human supervisory control behavior. In Proceedings of the AIAA Infotech@ Aerospace Conference and AIAA Unmanned Unlimited Conference, Washington, DC, USA, 6-9 April 2009; p. 1842.

6. Reason, J. Human error: Models and management. BMJ 2000, 320, 768-770. [CrossRef] [PubMed]

7. Cacciabue, P.C. Human error risk management for engineering systems: A methodology for design, safety assessment, accident investigation and training. Reliab. Eng. Syst. Saf. 2004, 83, 229-240. [CrossRef]

8. Lin, B. Behavior of ship officers in maneuvering to prevent a collision. J. Mar. Sci. Technol. 2006, 14, $225-230$.

9. Grant, E.; Salmon, P.M.; Stevens, N.J.; Goode, N.; Read, G.J. Back to the future: What do accident causation models tell us about accident prediction? Saf. Sci. 2018, 104, 99-109. [CrossRef]

10. Wood, D.D.; Dekker, S.; Cook, R.; Johannesen, L.; Sarter, N. Behind Human Error; CRC Press: Boca Raton, FL, USA, 2017.

11. KMST. Available online: https://data.kmst,go.kr/kmst/verdict/writtenverdict/selectwrittenverdit.do (accessed on 5 May 2019).

12. Branch, M.A.I.; House, C.; Place, C. Bridge Watchkeeping Safety Study; Department for Transportation, Marine Accident Investigation Branch: Southampton, UK, 2004; Volume 28.

13. Corporate, A.G. Safety and Shipping Review; Allianz Global Corporate and Specialty: Hong Kong, China, 2018.

14. Chauvin, C.; Lardjane, S.; Morel, G.; Clostermann, J.P.; Langard, B. Human and organisational factors in maritime accidents: Analysis of collisions at sea using the HFACS. Accid. Anal. Prev. 2013, 59, $26-37$. [CrossRef] [PubMed]

15. Murai, K.; Hayashi, Y.; Stone, L.C.; Inokuchi, S. Basic evaluation of performance of bridge resource teams involved in on-board smart education: Lookout pattern. In Review of the Faculty of Maritime Sciences; Kobe University: Kobe, Japan, 2006; Volume 3, pp. 77-83.

16. Mohovic, D.; Mohovic, R.; Baric, M. Deficiencies in learning COLREGs and new teaching methodology for nautical engineering students and seafarers in lifelong learning programs. J. Navig. 2016, 69, 765-776. [CrossRef]

17. Cordon, J.R.; Mestre, J.M.; Walliser, J. Human factors in seafaring: The role of situation awareness. Saf. Sci. 2017, 93, 256-265. [CrossRef]

18. Sandhåland, H.; Oltedal, H.; Eid, J. Situation awareness in bridge operations-A study of collisions between attendant vessels and offshore facilities in the North Sea. Saf. Sci. 2015, 79, 277-285. [CrossRef]

19. Youn, I.H.; Park, D.J.; Yim, J.B. Analysis of lookout activity in a simulated environment to investigate maritime accidents caused by human error. Appl. Sci. 2019, 9, 4. [CrossRef]

20. Kongsberg K-SIM NAVIGATION. Available online: https://www.kongsberg.com/digital/products/maritimesimulation/k-sim-navigation (accessed on 27 May 2019).

21. Dupont, W.D.; Plummer, W.D., Jr. Power and sample size calculations: A review and computer program. Control. Clin. Trials 1990, 11, 116-128. [CrossRef]

22. IMO. Convention on the International Regulations for Preventing Collisions at Sea, [with Amendments Adopted from December 2009]; International Maritime Organization: London, UK, 1972.

23. Park, D.J.; Kim, H.T.; Yang, H.S.; Yim, J.B. Comparisons of seafarers' perception of maritime and onshore traffic condition. J. Korean Soc. Mar. Environ. Saf. 2019, 25, 320-327. [CrossRef]

24. Gould, K.S.; Røed, B.K.; Saus, E.R.; Koefoed, V.F.; Bridger, R.S.; Moen, B.E. Effects of navigation method on workload and performance in simulated high-speed ship navigation. App. Ergon. 2009, 40, 103-114. [CrossRef] [PubMed]

25. Goerlandt, F.; Montewka, J. Maritime transportation risk analysis: Review and analysis in light of some foundational issues. Reliab. Eng. Syst. Saf. 2015, 138, 115-134. [CrossRef] 
26. Ahn, J.H.; Rhee, K.P.; You, Y.J. A study on the collision avoidance of a ship using neural networks and fuzzy logic. App. Ocean Res. 2012, 37, 162-173. [CrossRef]

27. IMO. IMO Standard Marine Communication Phrases (SMCP) [Sub-Committee on Safety of Navigation]; NAV 46/INF.4.; International Maritime Organization: London, UK, 2000.

28. Yim, J.B. Development of collision risk evaluation model between passing vessel and Mokpo Harbour Bridge. J. Navig. Port Res. 2010, 34, 405-415. [CrossRef]

29. Yim, J.B.; Kim, D.H. Statistical parameter estimation to calculate collision probability between Mokpo Harbor Bridge and passing vessels. J. Navig. Port Res. 2010, 34, 609-614. [CrossRef]

30. Gang, S.G.; Jeong, J.Y.; Yim, J.B. Applications of ship domain theory to identify risky sector in VTS area. J. Korean Soc. Mar. Environ. Saf. 2014, 20, 277-284. [CrossRef]

31. Goodwin, E.M. A statistical study of ship domains. J. Navig. 1975, 28, 328-344. [CrossRef]

32. Davis, P.V.; Dove, M.J.; Stockel, C.T. A computer simulation of marine traffic using domains and arenas. J. Navig. 1980, 33, 215-222. [CrossRef]

33. Zezhao, H.; Hongyan, X.; Guoyan, Z.; Jinfen, L.; Huimin, H.; Wenxiang, D. Analyses of thermal stress and fracture during cryopreservation of blood vessel. Sci. China Ser. E Technol. Sci. 2001, 44, 158-163.

34. Gitterman, D.P.; Evans, R.J. Nerve evoked P2X receptor contractions of rat mesenteric arteries; dependence on vessel size and lack of role of L-type calcium channels and calcium induced calcium release. Br. J. Pharmacol. 2001, 132, 1201-1208. [CrossRef] [PubMed]

35. Sang, L.Z.; Yan, X.P.; Wall, A.; Wang, J.; Mao, Z. CPA calculation method based on AIS position prediction. J. Navig. 2016, 69, 1409-1426. [CrossRef]

36. Rabiner, L.R.; Juang, B.H. An introduction to hidden Markov models. IEEE ASSP Mag. 1986, 3, 4-16. [CrossRef]

37. Mccallum, A. Hidden Markov models Baum welch algorithm. In Introduction to Natural Language Processing CS585; University of Massachusetts Amherst: Massachusetts, USA, 2004.

38. Nava, A.; Garrido, L.; Brena, R.F. Recognizing activities using a kinect skeleton tracking and hidden Markov models. In Proceedings of the 2014 13th Mexican International Conference on Artificial Intelligence, Tuxtla Gutierrez, Mexico, 16-22 November 2014; pp. 82-88.

39. Viterbi, A. Error bounds for convolutional codes and an asymptotically optimum decoding algorithm. IEEE Trans. Inf. Theory 1967, 13, 260-269. [CrossRef]

40. Forney, G.D. The viterbi algorithm. Proc. IEEE 1973, 61, 268-278. [CrossRef]

41. Krogh, A. Two methods for improving performance of an HMM application for gene finding. Cent. Biol. Seq. Anal. Phone 1997, 45, 4525.

42. Baum, L. An inequality and associated maximization technique in statistical estimation of probabilistic functions of a Markov process. Inequalities 1972, 3, 1-8.

43. Rabiner, L.R. A tutorial on hidden Markov models and selected applications in speech recognition. Proc. IEEE 1989, 77, 257-286. [CrossRef]

44. Zimmerman, G. Modeling and simulation of individual user behavior for building performance predictions. In Proceedings of the 2007 Summer Computer Simulation Conference, San Diego, CA, USA, 16-19 July 2007; Society for Computer Simulation International: San Diego, CA, USA, 2007; pp. 913-920.

45. Lee, C.K.; Moon, S.B.; Jeong, T.G. The investigation of ship maneuvering with hydrodynamic effects between ships in curved narrow channel. Int. J. Nav. Arch. Ocean 2016, 8, 102-109. [CrossRef]

46. Inoue, K. Evaluation method of ship-handling difficulty for navigation in restricted and congested waterways. J. Navig. 2000, 53, 167-180. [CrossRef]

47. Yim, J.B.; Kim, D.S.; Park, D.J. Modeling perceived collision risk in vessel encounter situations. Ocean Eng. 2018, 166, 64-75. [CrossRef]

48. Chin, H.C.; Debnath, A.K. Modeling perceived collision risk in port water navigation. Saf. Sci. 2009, 47, 1410-1416. [CrossRef]

49. Kozak, J.J.; Hancock, P.A.; Arthur, E.J.; Chrysler, S.T. Transfer of training from virtual reality. Ergonomics 1993, 36, 777-784. [CrossRef]

50. Perez, T. Ship Motion Control: Course Keeping and Roll Stabilisation Using Rudder and Fins; Springer Science \& Business Media: Berlin, Germany, 2006. 
51. Embrey, D. Understanding human behaviour and error. Hum. Reliab. Assoc. 2005, 1, 1-10.

52. Yim, J.B. A study on the analysis and identification of seafarers' skill-rule-knowledge inherent in maritime accidents. J. Korean Soc. Mar. Environ. Saf. 2017, 23, 224-230. [CrossRef]

(C) 2019 by the authors. Licensee MDPI, Basel, Switzerland. This article is an open access article distributed under the terms and conditions of the Creative Commons Attribution (CC BY) license (http://creativecommons.org/licenses/by/4.0/). 